\title{
Efectos del cambio climático sobre la oferta y precios de cebolla de bulbo. Caso del municipio Duitama, Boyacá (Colombia)
}

\section{Climatic change effects on the supply and prices of bulb onion. Case of Duitama municipality, Boyacá (Colombia)}
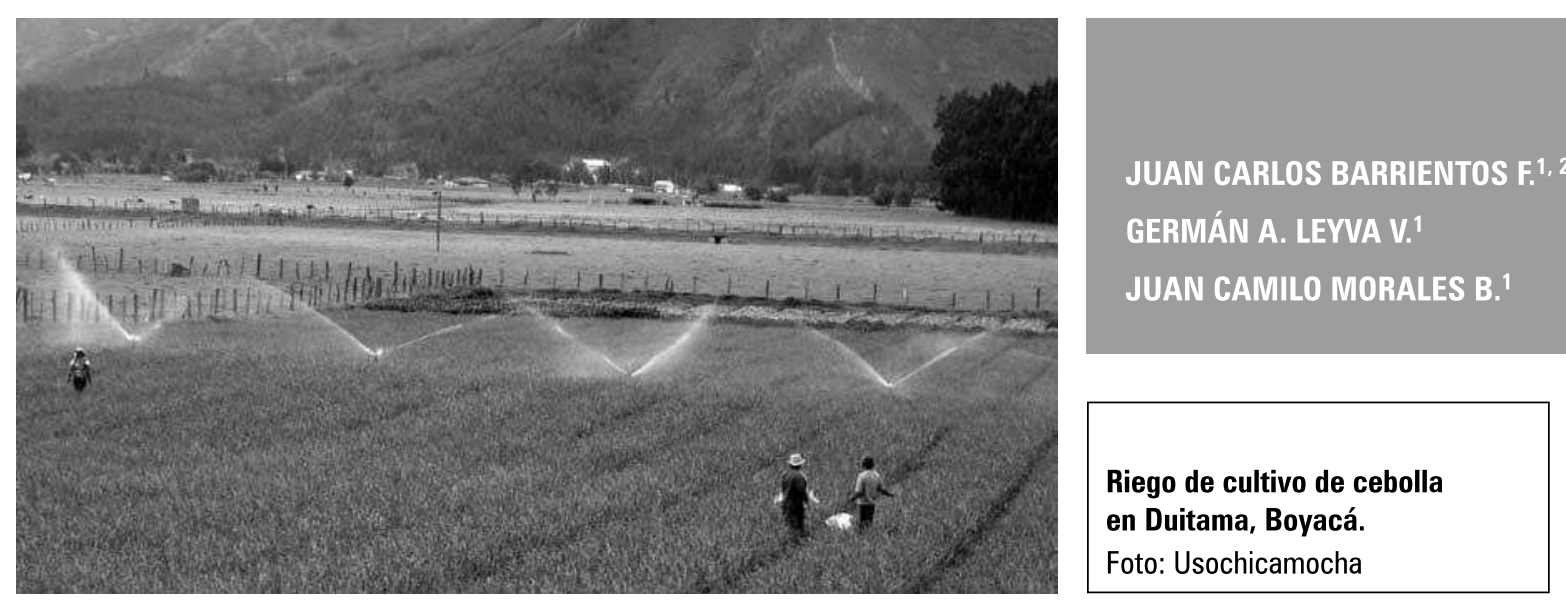

\section{RESUMEN}

La cebolla (Allium cepa L.) es la segunda hortaliza más consumida en Colombia. La producción y los precios de mercado de esta hortaliza están determinados por varios factores, entre ellos el clima. Últimamente, el clima ha experimentado cambios en su comportamiento. Estos cambios están afectando, entre otros, la productividad agraria. En el caso de la cebolla, se presume que el cambio climático está afectando tanto la producción, como la oferta y los precios de la cebolla de bulbo. En ese sentido, el objetivo de esta investigación es determinar el modo en que el cambio climático está afectando la producción y precios de la cebolla de bulbo, basado en un estudio de caso. Se analizaron datos climáticos de la zona de estudio, distrito de riego Alto Chicamocha en Duitama, Boyacá, y datos de producción y precios del departamento y del país. Se realizaron entrevistas con productores y un experto de la zona en cuestión. Con esta información se ha llegado a los siguientes resultados y conclusiones: el cambio climático es un fenómeno presente en la zona de estudio, caracterizado, entre otros, por la disminución del brillo solar y de la humedad relativa, incremento de la precipitación, evaporación y temperatura; sus efectos han determinado cambios en el manejo del cultivo, como incremento de dosis y frecuencia de aplicación de fungicidas principalmente, pero no han incidido de manera determinante en la cantidad de producción (oferta local); los precios de la cebolla son afectados por la importación y las expectativas (de mayor ganancia) de los productores más que por el cambio climático.

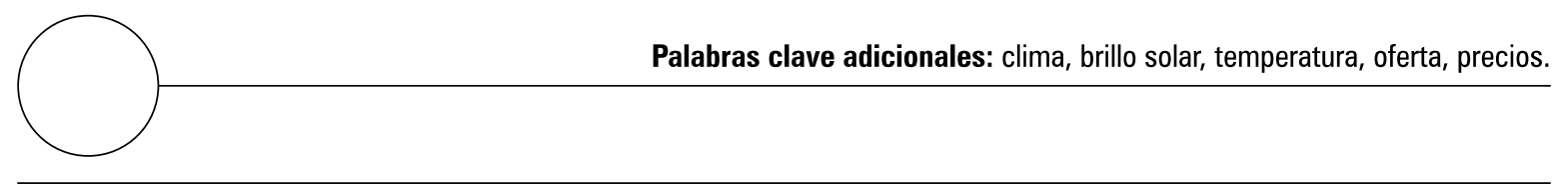

Departamento de Agronomía, Facultad de Agronomía, Universidad Nacional de Colombia, Bogotá (Colombia).

2 Autor para correspondencia. jcbarrientosf@unal.edu.co 


\section{ABSTRACT}

Onion (Allium cepa L.) is the second vegetable most consumed in Colombia. The production and the market prices of this vegetable are determined by many factors, among them, the climate. Lately, the climate has undergone changes in behavior. These changes are affecting, among others, the agrarian productivity. In onion case, it is presumed that the climatic change is affecting so much the production as the supply and the prices of the bulb onion. In that sense, the objective of this research is, based on a case study, to determine the way in that the climatic change is affecting the production and prices of bulb onion. Climatic data of the study zone, irrigation district of Alto Chicamocha in Duitama, Boyacá, have been analyzed, as well as production and prices data of the department and the country. Interviews with producers and the expert of the zone have been realized. With this information the research has reached the following results and conclusions: the climatic change is a present phenomenon in the zone of study, characterized, among others, by the diminution of solar brightness and relative humidity, an increase of precipitation, evaporation and temperature; their effects have determined changes in crop management, such as increase of dose and frequency of application of fungicides mainly, but these have not affected in a determent form the amount of production (local supply); the onion prices are affected by the import and the expectations (of greater gain) of the producers more than by the climatic change.

Additional keywords: climate, solar intensity, temperature, supply, prices.

Fecha de recepción: 11-05-2009

Aprobado para publicación: 01-06-2009

INTRODUCCIÓN

\section{Oferta de cebolla en Colombia}

La cebolla (Allium cepa L.) es la segunda hortaliza más consumida en Colombia. Según la CCI (2003), el consumo per cápita nacional de cebolla (junca o larga y de bulbo o cabezona) es de $6 \mathrm{~kg} /$ persona-año, mostrándose un incremento aproximado del 2,7\% entre 1997 y 2001 (Agronet, 2009). La cebolla que se vende en Colombia proviene principalmente de la oferta nacional, aunque en los últimos años la oferta de cebolla importada ha incrementado sus volúmenes, de 11.028 t en 1997 a 71.826 t en 2008 (Agronet, 2009). La producción nacional, que en 1997 era de 897.199 t, ha tenido un descenso considerable, tal que para 2008 fue de 568.457 t. Este descenso se debió principalmente a la disminución de la producción anual de cebolla junca, la que bajó de 666.604 t en 1997 a 291.305 t en 2008, mientras que la producción anual de cebolla de bulbo se mantuvo oscilando entre 200.000 (1998) y 300.000 t (2007) aproximadamente, con una clara tendencia de incremento de cerca del 3\% anual. La superficie dedicada a la producción de cebolla en Colombia en los últimos 12 años ha oscilado alrededor de 20.000 ha, según las estadísticas 26.714 ha en 1997, 16.286 ha en 2002 y 31.384 ha en 2008 (Agronet, 2009). Del mismo modo que la producción, la superficie nacional de cultivo de cebolla junca ha disminuido de 15.110 ha en 1997 a 14.868 ha en 2008; mientras que el cultivo de cebolla de bulbo se ha incrementado paulatinamente, de 11.604 ha en 1997 a 16.516 ha en 2008 (Agronet, 2009). Por otra parte, el rendimiento de cebolla de bulbo en los últimos 12 años se ha mantenido alrededor de 20 t ha1; mientras que el de cebolla junca ha bajado de 44,1 t ha-1 en 1997 a 19,5 t ha $a^{-1}$ en 2008 (Agronet, 2009). Actualmente, son siete los departamentos donde se cultiva cebolla de bulbo, de los cuales, para el año 2008, Boyacá aportó con 49\% de la producción nacional, Norte de Santander con $23 \%$ y Cundinamarca 20\%. La superficie de cultivo en Boyacá osciló notablemente, por ejem- 
plo en 1998 fue de 3.686 ha, en 2001 de 4.005 ha, en 2002 de 5.553 ha, en 2007 de 6.149 ha y en 2008 de 4.262 ha. Por el contrario, en Norte de Santander la superficie de cultivo ha tenido un incremento sostenido de aproximadamente un $14 \%$ anual. Entretanto, también Cundinamarca incrementó su superficie de cultivo, pero recién a partir de 2005 , en aproximadamente un $44 \%$ anual. Boyacá, aunque con oscilaciones de casi 5 $\mathrm{t} \mathrm{ha}^{-1}$, ha venido incrementando sus rendimientos, por ejemplo de $24 \mathrm{tha}^{-1}$ en 1998 a $28 \mathrm{t} \mathrm{ha}^{-1} \mathrm{el}$ 2006 y a 32 t ha ${ }^{-1}$ el 2008. Por el contrario, Norte de Santander, con un promedio aproximado de $21 \mathrm{t} \mathrm{ha}^{-1}$, y Cundinamarca, con un promedio aproximado de $17 \mathrm{t} \mathrm{ha}^{-1}$, han experimentado en los últimos dos años descensos notables (6 a $10 \mathrm{t}$ ha $^{-1}$ ) en sus rendimientos (Agronet, 2009).

\section{Factores determinantes de la oferta de cebolla}

Los principales factores que determinan la oferta de cebolla de bulbo, como en otros cultivos, son, entre otros, los precios del producto, los costos de los factores de producción, efectos climáticos y fitosanitarios y la tecnología de producción.

\section{Precios y costos de producción de la cebolla}

Los precios del producto incentivan o desaniman a los productores, a su vez, la cantidad de oferta en el mercado determina la subida o bajada de precios. En el caso de la cebolla de bulbo, los precios promedio en Corabastos (Bogotá) correspondientes a los años 1997 (511 \$/kg) hasta 2002 (792 \$/kg) muestran un comportamiento más o menos regular de bajadas y subidas, atribuible al efecto del teorema de la telaraña (Ezekiel, 1938), después del cual se advierten oscilaciones leves hasta 2007 y luego un incremento notorio entre 2007 (831 \$/kg) y 2008 (1063\$/kg). Según datos del Banco Agrario de Colombia (2008), el costo de producción desde semillero de una hectárea de cebolla de bulbo (cabezona) en Boyacá asciende a cerca de diez millones de pesos, de los cuales aproximadamente un 15\% corresponde a aplicación de riego, un 10\% a semilla (Agrocadenas, 2005 , reporta un $23 \%$ ), un $10 \%$ a fertilizantes, un $3 \%$ a control de plagas y enfermedades, un $1,5 \%$ a fungicidas y un $0,5 \%$ a fertilización. El gasto en mano de obra abarca cerca del $50 \%$ de los costos totales de producción, de los cuales el riego, la cosecha y siembra (trasplante) son las labores de mayor demanda de trabajo.

\section{Factores fitosanitarios}

Si bien el costo del control de enfermedades y de fungicidas es porcentualmente bajo (3-5\%), su importancia en el proceso de producción de cebolla es mayor, ya que de ello depende, en gran medida, el éxito o fracaso del negocio. Dos enfermedades son especialmente restrictivas para el cultivo de cebolla: el tizón (Peronospora destructor) y la alternaria (Alternaria porri) (Agrios, 2005; Maroto et al., 1995). Su control depende tanto del manejo del cultivo como de las condiciones climáticas de la zona y periodo del año.

\section{Cambio climático y producción de cebolla}

El fenómeno del cambio climático, expresado a través de cambios y variaciones en las temperaturas, precipitaciones y concentraciones de $\mathrm{CO}_{2}$ en la atmósfera, está afectando, entre otros, la productividad agraria, especialmente de las zonas tropicales del planeta (Kurukulasuriya y Rosenthal, 2003). En el caso de la producción de cebolla, los cambios en la forma, la cantidad, la frecuencia e intensidad de las precipitaciones han determinado un nuevo régimen de humedad del suelo y del aire que, sumado a los cambios en las variaciones diarias, mensuales y anuales de la temperatura, ha creado un nuevo ambiente, muchas veces más favorable, para la proliferación de enfermedades (Brewster, 1997).

\section{PLANTEAMIENTO DEL PROBLEMA}

La producción de cebolla depende, entre otros, de los factores climáticos. La precipitación determina en el cultivo de cebolla de bulbo la cantidad y frecuencia de riego o drenaje. La luminosidad (horas luz) influye en la calidad y tamaño del 
bulbo. La humedad relativa y la temperatura, así como sus oscilaciones, determinan las condiciones favorables o desfavorables para enfermedades que atacan a la especie en estudio. Este escenario hace suponer que también en Colombia la producción de cebolla, y por ende la oferta y precios del producto, está siendo afectada por el cambio climático. Para confirmar o rechazar el supuesto mencionado, se ha tomado como objeto de estudio una zona productora de cebolla en Boyacá, la zona del distrito de riego Alto Chicamocha.

\section{Objetivo general}

Determinar los efectos que produce el cambio climático en los cultivos de cebolla de bulbo en la región del Alto Chicamocha (Boyacá).

\section{Objetivos específicos}

- Determinar las características del cambio climático en la zona.

- Determinar las manifestaciones del cambio climático y sus efectos en la producción de cebolla de bulbo en el distrito de riego Alto Chicamocha.

- Determinar las medidas adoptadas por los productores para contrarrestar los efectos del cambio climático en la producción de cebolla de bulbo.

- Determinar los efectos de la producción en la oferta (producción nacional e importación) y precios de la cebolla de bulbo.

\section{MÉTODO}

Esta investigación es, en una proporción mayor, de tipo exploratoria, sobre todo cuando se trata de la información primaria. Sin embargo, la información secundaria permite hacer análisis descriptivos y en algunos casos causales.

\section{Zona de estudio}

El distrito de Riego y Drenaje de Gran Escala Alto Chicamocha y Firavitoba abarca los municipios de Paipa, Duitama, Santa Rosa, Tibasosa, Nobsa, Sogamoso y Firavitoba. Tiene una superficie bruta de cubrimiento de 11.300 ha, un área de drenaje de 9.258 ha y un área de infraestructura de riego de 7.335 ha. Sus usuarios beneficiarios son 6.200 , de los cuales el $76,7 \%$ tiene parcelas agrarias menores a $1 \mathrm{ha}, 18,5 \%$ tiene parcelas entre 1 y 5 ha, 3,23\% tiene parcelas entre 5 y 10 ha y sólo 1,37\% tiene parcelas mayores a 10 ha. El área dedicada a producción agrícola es de 2.500 ha y a producción pecuaria, principalmente lechería, 6.758 ha. Según Usochicamocha (2008), en la zona se han destinado 2.300 ha a la producción de cebolla de bulbo, 12 ha para brócoli, 10 ha para coliflor, 70 ha para arveja y 35 ha para repollo. Para el presente estudio se ha considerado solamente el municipio Duitama y en éste la zona productora de cebolla de bulbo.

\section{Tipo de información y su obtención}

La información requerida para este trabajo fue la siguiente:

- Información mensual de los parámetros climáticos desde 1990 hasta 2008: brillo solar, evaporación, humedad relativa, precipitación, precipitación en 24 h, número de días con lluvia, temperatura media, temperatura máxima y temperatura mínima. Esta información, procedente de la estación Surbatá-Bonza del municipio Duitama, se obtuvo del Ideam (2009).

- Información de oferta de cebolla en Colombia desde 1997 hasta 2008: superficie de producción, producción anual, rendimiento e importación anual por países (Agronet, 2009).

- Información de precios promedio mensual de mayoristas de cebolla de bulbo desde 1997 hasta 2008 (Corabastos, 2009; CCI, 2009). 
- Información primaria sobre el efecto del cambio climático en la producción de cebolla. Esta información se obtuvo de seis productores de cebolla "representativos" del municipio Duitama a través de entrevistas. Las entrevistas, con una duración aproximada de 25 a $40 \mathrm{~min}$, se realizaron el 25 de junio de 2009, con base en un cuestionario de 20 preguntas abiertas. El cuestionario contenía, entre otros, los temas: presencia-ausencia del cambio climático en la zona, manifestación del cambio climático, efectos del cambio climático en la producción de cebolla, medidas adoptadas para contrarrestar el efecto del cambio climático, factores que influyen en la fijación de precios de la cebolla, efectos del cambio climático en el precio de la cebolla y algunas características de las fincas productoras de cebolla de bulbo de la zona de estudio. Paralelamente, se entrevistó al jefe del Departamento de Planeación y Desarrollo Productivo del Distrito de Riego para tratar temas relativos a la producción de cebolla: superficie, producción, rendimientos, problemas más importantes, etc. La entrevista, con una duración de aproximadamente $3 \mathrm{~h}$, se llevó a cabo mediante un cuestionario no estructurado con preguntas abiertas.

\section{Análisis de la información}

\section{Información mensual y anual de los parámetros climáticos}

- Descripción de las características climáticas de la zona de estudio con base en datos anuales acumulados o promedio.

- Determinación de la regularidad o irregularidad de ocurrencia de los eventos climáticos.

\section{Información de oferta de cebolla en Colombia}

- Análisis de la producción nacional.

- Relación de la producción con los parámetros de clima.
- Análisis de la importación de la cebolla.

- Comparación de la oferta nacional e importación de cebolla con los precios nacionales.

Información de precios promedio mensual de mayoristas de cebolla de bulbo

- Análisis comparativo de los precios por meses.

- Análisis comparativo de los precios por años.

- Relación de los precios con la oferta nacional e importación de cebolla de bulbo.

- Relación de los precios con algunos factores climáticos.

\section{Información primaria sobre el efecto del cambio climático en la producción de cebolla}

- Análisis de los efectos del cambio climático en la producción de cebolla.

- Análisis de las medidas adoptadas por los productores para contrarrestar el efecto del cambio climático en la producción de cebolla.

- Análisis de los efectos del cambio climático en los precios de la cebolla.

\section{RESULTADOS Y DISCUSIÓN}

\section{Características del cambio climático en la zona}

De los datos climáticos presentados en la tabla 1, lo que más se destaca, caracterizando a la zona, es lo siguiente:

- En promedio, hay $5 \mathrm{~h}$ diarias de brillo solar. Esto podría ser restrictivo para variedades de cebolla de bulbo que requieren de más horas luz para desarrollo foliar o llenado de bulbo. 
Tabla 1. Principales datos climáticos anuales de la estación Surbatá-Bonza del municipio Duitama, Boyacá (1990-2008).

\begin{tabular}{|c|c|c|c|c|c|c|c|c|c|c|c|c|c|c|c|c|c|c|}
\hline \multirow[t]{2}{*}{ Años } & \multicolumn{2}{|c|}{ Brillo solar } & \multicolumn{2}{|c|}{ Evaporación } & \multicolumn{2}{|c|}{$\begin{array}{c}\text { Humedad relativa } \\
(\%)\end{array}$} & \multicolumn{2}{|c|}{ Precipitación } & \multicolumn{2}{|c|}{$\begin{array}{c}\text { Precipitación } \\
\text { máxima en } \\
24 \mathrm{~h} \\
\end{array}$} & \multicolumn{2}{|c|}{$\begin{array}{c}\text { No. de días } \\
\text { con lluvia/año }\end{array}$} & \multicolumn{2}{|c|}{$\begin{array}{c}\text { Temperatura } \\
\text { media }\left({ }^{\circ} \mathrm{C}\right) \\
\end{array}$} & \multicolumn{2}{|c|}{$\begin{array}{l}\text { Temperatura } \\
\text { máxima }\left({ }^{\circ} \mathrm{C}\right)\end{array}$} & \multicolumn{2}{|c|}{$\begin{array}{l}\text { Temperatura } \\
\text { mínima }\left({ }^{\circ} \mathrm{C}\right) \\
\end{array}$} \\
\hline & $\left(\mathrm{h}\right.$ año $\left.{ }^{-1}\right)$ & Rango* & $\left(m m\right.$ año $\left.{ }^{-1}\right)$ & Rango* & $\begin{array}{l}\text { Promedio } \\
\text { anual }\end{array}$ & Rango* & $\left(\mathrm{mm}\right.$ año $\left.0^{-1}\right) \mid$ & Rango* & $(\mathrm{mm})$ & Rango* & Anual & Rango* & Anual & Rango* & Anual & Rango* & Anual & Rango* \\
\hline 1990 & 1.715 & 48 & 1.171 & 47 & 80 & 11 & 872 & 198 & 49 & 43 & 166 & 14 & 14 & 1 & 24 & 2 & -1 & 5 \\
\hline 1991 & 1.831 & 68 & 1.272 & 30 & 79 & 18 & 760 & 150 & 23 & 18 & 157 & 17 & 14 & 2 & 26 & 4 & -3 & 7 \\
\hline 1992 & 1.726 & 83 & 1.261 & 52 & 80 & 10 & 556 & 88 & 32 & 30 & 139 & 15 & 14 & 2 & 27 & 5 & -2 & 7 \\
\hline 1993 & 1.838 & 75 & 1.218 & 31 & 84 & 11 & 961 & 180 & 32 & 26 & 188 & 20 & 14 & 2 & 24 & 2 & -3 & 8 \\
\hline 1994 & 1.702 & 111 & 1.169 & 40 & 78 & 25 & 895 & 153 & 32 & 31 & 195 & 22 & 14 & 2 & 25 & 4 & -1 & 6 \\
\hline 1995 & 1.782 & 83 & 1.201 & 43 & 78 & 15 & 887 & 127 & 27 & 18 & 201 & 21 & 14 & 2 & 28 & 7 & -4 & 10 \\
\hline 1996 & 1.784 & 103 & 1.208 & 40 & 80 & 8 & 918 & 102 & 32 & 23 & 202 & 14 & 14 & 2 & 24 & 3 & -1 & 7 \\
\hline 1997 & 1.967 & 116 & 1.399 & 44 & 75 & 9 & 669 & 98 & 36 & 29 & 147 & 17 & 14 & 1 & 25 & 3 & -2 & 7 \\
\hline 1998 & 1.782 & 87 & 1.389 & 65 & 77 & 13 & 964 & 196 & 54 & 54 & 199 & 29 & 15 & 3 & 25 & 2 & 0 & 6 \\
\hline 1999 & 1.740 & 98 & 1.247 & 63 & 80 & 7 & 1.091 & 130 & 32 & 25 & 206 & 15 & 14 & 1 & 28 & 6 & 1 & 5 \\
\hline 2000 & 1.884 & 130 & 1.225 & 47 & 79 & 7 & 856 & 104 & 33 & 28 & 184 & 15 & 14 & 1 & 24 & 2 & -1 & 6 \\
\hline 2001 & 1.973 & 109 & 1.398 & 43 & 69 & 11 & 611 & 104 & 25 & 20 & 158 & 17 & 14 & 2 & 26 & 3 & -3 & 8 \\
\hline 2002 & 1.885 & 129 & 1.408 & 44 & 74 & 12 & 719 & 132 & 31 & 28 & 152 & 15 & 14 & 2 & 25 & 3 & 0 & 6 \\
\hline 2003 & 1.793 & 155 & 1.347 & 56 & 81 & 14 & 1.053 & 225 & 42 & 40 & 173 & 22 & 14 & 1 & 29 & 6 & 1 & 5 \\
\hline 2004 & 1.968 & 95 & 1.223 & 25 & 86 & 7 & 906 & 175 & 56 & 51 & 158 & 21 & 15 & 1 & 29 & 6 & -4 & 10 \\
\hline 2005 & 1.811 & 106 & 1.311 & 63 & 83 & 5 & 904 & 161 & 37 & 29 & 172 & 16 & 15 & 1 & 25 & 3 & 1 & 6 \\
\hline 2006 & 1.611 & 118 & 1.256 & 26 & 81 & 17 & 1.003 & 139 & 33 & 26 & 180 & 15 & 15 & 3 & 24 & 4 & 0 & 6 \\
\hline 2007 & 1.578 & 134 & 1.216 & 54 & 76 & 9 & 934 & 124 & 39 & 31 & 166 & 17 & 15 & 2 & 26 & 2 & -8 & 13 \\
\hline 2008 & 1.697 & 98 & 1.240 & 57 & 77 & 7 & 882 & 142 & 36 & 29 & 171 & 18 & 15 & 1 & 27 & 4 & 0 & 5 \\
\hline Promedio & 1.793 & 102 & 1.271 & 46 & 79 & 11 & 865 & 144 & 36 & 30 & 174 & 18 & 14 & 2 & 26 & 5 & -2 & 7 \\
\hline
\end{tabular}

Fuente: Elaborado con base en datos del Ideam, 2009.

* Diferencia entre el mes del año con el valor más alto y el mes del año con el valor más bajo. 
- La evaporación es, en promedio, mayor a la precipitación en aproximadamente $400 \mathrm{~mm}$. Esto significa, de manera general, que para la producción vegetal se necesita un aporte complementario de agua al suelo.

- Cerca de la mitad del tiempo está lloviendo, lo que favorece el mantenimiento de la humedad del ambiente.

- La humedad del ambiente es relativamente elevada. Esta es una condición favorable para la proliferación de enfermedades en el cultivo de la cebolla.

- Las precipitaciones en 24 h están alrededor de $36 \mathrm{~mm}$. Esto podría significar, en algunos casos, encharcamiento de las parcelas de cebolla creándose un ambiente favorable para el desarrollo de enfermedades del cultivo.

- Si bien la temperatura media se ha mantenido entre $14^{\circ} \mathrm{C}$ y $15^{\circ} \mathrm{C}$, el rango entre temperaturas máximas y mínimas es mayor a $25^{\circ} \mathrm{C}$. Esto puede ir en detrimento del cultivo de cebolla, ya que las temperaturas bajas reducen o detienen el crecimiento, pudiendo dañar también los tejidos de la planta. Por otra parte, las temperaturas mayores a $23^{\circ} \mathrm{C}$ favorecen el desarrollo de la planta, pero también de enfermedades del cultivo.
Mediante los "rangos", presentados en la tabla 1, se desea hacer notar el comportamiento regular o irregular de los parámetros climáticos durante el periodo de estudio. Teóricamente, si los eventos climáticos se repitieran y ocurrieran con regularidad, los rangos entre los valores máximos y mínimos mensuales del año serían similares en todos los casos. Sin embargo, la tendencia de los valores hallados es de incremento en la diferencia entre los extremos, es decir, los eventos climáticos se estarían haciendo más variables y extremos (tabla 1). Llaman la atención especialmente el brillo solar, la humedad relativa y las temperaturas máximas. Este fenómeno de mayor diferencia entre extremos dificultaría aún más la precisión en la planificación de la producción vegetal.

La tabla 2 muestra que, según las tendencias lineales, a largo plazo:

- Hay un incremento en la temperatura media, máxima y mínima. Esto podría favorecer al desarrollo del cultivo de cebolla, pero también a la proliferación de enfermedades.

- A consecuencia del incremento de temperatura, hay un incremento de la evaporación, lo que hace pensar en una mayor necesidad de riego para el cultivo. Sin embargo, también hay un

Tabla 2. Tendencia lineal de los principales parámetros climáticos de la estación Surbatá-Bonza del municipio Duitama, Boyacá (1990-2008).

\begin{tabular}{|c|c|c|c|c|c|c|c|c|c|}
\hline Ítem & $\begin{array}{l}\text { Brillo } \\
\text { solar }\end{array}$ & $\begin{array}{l}\text { Evapo- } \\
\text { ración }\end{array}$ & $\begin{array}{c}\text { Humedad } \\
\text { relativa }\end{array}$ & $\begin{array}{l}\text { Precipi- } \\
\text { tación }\end{array}$ & \begin{tabular}{|c|}
$\begin{array}{c}\text { Precipi- } \\
\text { tación } \\
\text { máxima en } \\
24 \text { horas }\end{array}$ \\
\end{tabular} & $\begin{array}{c}\text { No. } \\
\text { de días }\end{array}$ & $\begin{array}{l}\text { Tempe- } \\
\text { ratura } \\
\text { media } \\
\text { anual } \\
\end{array}$ & $\begin{array}{l}\text { Tempe- } \\
\text { ratura } \\
\text { máxima } \\
\text { anual } \\
\end{array}$ & $\begin{array}{c}\text { Tempe- } \\
\text { ratura } \\
\text { mínima } \\
\text { anual } \\
\end{array}$ \\
\hline & (h día ${ }^{-1}$ ) & $(\mathrm{mm}$ año-1) & $\begin{array}{l}\text { Promedio } \\
\text { anual (\%) }\end{array}$ & $(\mathrm{mm}$ año-1) & $(\mathrm{mm})$ & $\begin{array}{c}\text { con lluvia/ } \\
\text { año }\end{array}$ & $\left({ }^{\circ} \mathrm{C}\right)$ & $\left({ }^{\circ} \mathrm{C}\right)$ & $\left({ }^{\circ} \mathrm{C}\right)$ \\
\hline $\begin{array}{l}\text { Promedio } \\
\text { (1990-2008) }\end{array}$ & 5 & 1271 & 79 & 865 & 36 & 174 & 14 & 26 & -2 \\
\hline $\begin{array}{l}\text { Tendencia de } \\
\text { valores entre } 1990 \\
\text { y } 2008(\%)\end{array}$ & $-2,3$ & +5 & -1 & +16 & +17 & $-2,4$ & $+7,8$ & +5 & +21 \\
\hline $\begin{array}{l}\text { Tendencia de los } \\
\text { rangos }(\%)^{*}\end{array}$ & +73 & +19 & +40 & +1 & +13 & 0 & -25 & +42 & +14 \\
\hline
\end{tabular}

Fuente: Elaborado con base en datos del Ideam, 2009.

${ }^{*}$ Con base en los datos de la Tabla 1. 
incremento en la precipitación, y en mayor proporción que la evaporación, por lo que la necesidad de riego tendería a reducirse.

- Como efecto del incremento de la precipitación, las horas luz tienden a disminuir. Esto podría incidir negativamente en la ganancia de peso y calidad del bulbo de la cebolla.

- Hay un incremento en la intensidad de las lluvias y una disminución en su frecuencia, es decir, las lluvias serán más cortas, más fuertes y menos frecuentes, lo que podría llegar a ser menos favorable para la producción vegetal.

\section{Manifestaciones del cambio climático y sus efectos en la producción de cebolla de bulbo en el distrito de riego Alto Chicamocha}

Los productores entrevistados y sus parcelas de producción presentan características que se resumen a continuación:

Los productores entrevistados tienen basta experiencia en el cultivo de cebolla de bulbo. En atención a la superficie de cultivo, se diferencian en pequeños, medianos y grandes productores. Los rendimientos que obtienen en esta zona están entre los más altos del país, favorecidos, entre otros, por la disponibilidad de riego. La mayoría siembra cebolla dos veces al año, pero también hay agricultores que han escalonado las siembras, llegando a valores de hasta seis por año. Algo que llama la atención es la frecuencia de riego. Según el técnico entrevistado, los riegos se realizan rutinariamente (1-3 horas por riego) y en abundancia, siendo común ver parcelas casi encharcadas después de un riego. Dado que la mayoría de las propiedades agrícolas son pequeñas $(<1 \mathrm{ha})$, los productores de cebolla optan por arrendar o cultivar en compañía, así las parcelas de cebolla son itinerantes. Otro motivo para la itinerancia de las parcelas de cultivo de cebolla, según uno de los entrevistados, es la infección de los suelos (después de cuatro años seguidos de cultivo de cebolla) con el "tizón" (Peronospora destructor) y la "al- ternaria" (Alternaria porri) y la disponibilidad de riego. Es así que la mayoría de los productores de la zona de estudio proceden de otros municipios como: Samacá, Sáchica, Villa de Leyva, Sutamarchan, Tinjacá, Choachí y Cáqueza.

Todos los entrevistados manifestaron haber percibido cambios en el clima. Los cambios a los que hicieron referencia son los siguientes:

- El ambiente está más seco.

- La temperatura es mayor, hace más calor.

- Hay mayor presencia de niebla, por tanto más frío por las mañanas y menor brillo solar.

- Las lluvias son más irregulares. Las épocas de lluvia, que eran bien marcadas (abril-mayo; octubre-noviembre), ya no tienen la regularidad de antes.

- Los cambios de temperatura y lluvias son más bruscos e impredecibles.

La percepción de los productores entrevistados sobre el cambio climático coincide, en su mayoría, con las tendencias obtenidas de los datos del clima de la zona (tablas 1 y 2 ).

Aunque la superficie de cultivo no ha crecido considerablemente, Boyacá sigue siendo el mayor productor nacional de cebolla de bulbo (tabla 4). A su vez, el distrito de riego Alto Chicamocha concentra la mayor superficie de producción en el departamento, aproximadamente 2.500 ha (Usochicamocha, 2008); adicionalmente, los mayores rendimientos se presentan en esta zona, aproximadamente $40 \mathrm{t} \mathrm{ha}^{-1}$ (obtenido de tabla 3).

Si bien el clima tiene un efecto determinante en la producción agrícola, sus variables no han influido significativamente en el comportamiento de la producción, como lo muestra la tabla 5, excepto el de evaporación. Los factores climáticos determinantes del cultivo están siendo controla- 
Tabla 3. Características de los productores y de las unidades de producción de cebolla de bulbo en la zona de estudio.

\begin{tabular}{c|c|c|c|c|c|c|}
\hline $\begin{array}{c}\text { Experiencia } \\
\text { en el cultivo }\end{array}$ & $\begin{array}{c}\text { Cantidad } \\
\text { de semilla }\end{array}$ & $\begin{array}{c}\text { Superficie } \\
\text { parcela } \\
\text { de ceholla }\end{array}$ & Rendimiento & $\begin{array}{c}\text { Número de } \\
\text { siembras }\end{array}$ & $\begin{array}{c}\text { Frecuencia } \\
\text { de riego }\end{array}$ & $\begin{array}{c}\text { Tenencia } \\
\text { de tierra }\end{array}$ \\
\hline (años) & (lb/fanegada) & (fanegadas) & $\begin{array}{c}\text { (bultos/ } \\
\text { fanegada) }\end{array}$ & al año & (No./semana) & Aue maneja \\
\hline $5-20$ & $3-4$ & $1-70$ & $500-560$ & $2-6{ }^{2}$ & $1-3$ & $\begin{array}{c}\text { Arriendo, en } \\
\text { compañía }\end{array}$ \\
\hline
\end{tabular}

Fuente: Entrevista a productores de cebolla de bulbo del Distrito de Riego Alto Chicamocha (junio, 2009).

${ }^{1}$ Bulto $=50 \mathrm{~kg}$; fanegada $=6.400 \mathrm{~m}^{2} ;{ }^{2}$ En siembras escalonadas.

\begin{tabular}{|c|c|c|c|c|c|c|c|c|c|c|c|c|}
\hline Variable & 1997 & 1998 & 1999 & 2000 & 2001 & 2002 & 2003 & 2004 & 2005 & 2006 & 2007 & 2008 \\
\hline $\begin{array}{l}\text { Producción } \\
\text { Boyacá (t) }\end{array}$ & 95.894 & 88.391 & 127.656 & 142.436 & 104.793 & 95.500 & 110.889 & 117.272 & 113.803 & 133.351 & 151.727 & 136.770 \\
\hline $\begin{array}{l}\text { Producción } \\
\text { Colombia (t) }\end{array}$ & 230.595 & 203.410 & 257.099 & 276.300 & 224.945 & 216.558 & 232.817 & 251.752 & 253.728 & 294.790 & 312.673 & 277.152 \\
\hline $\begin{array}{l}\text { Superficie } \\
\text { cosechada } \\
\text { Boyacá (ha) }\end{array}$ & 3.921 & 3.686 & 4.913 & 5.553 & 4.005 & 4.094 & 4.734 & 4.290 & 4.328 & 4.798 & 6.149 & 4.262 \\
\hline $\begin{array}{l}\text { Superficie } \\
\text { cosechada } \\
\text { Colombia (ha) }\end{array}$ & 11.604 & 10.298 & 11.766 & 12.424 & 10.330 & 10.250 & 11.058 & 11.642 & 11.838 & 13.870 & 15.858 & 16.516 \\
\hline $\begin{array}{l}\text { Rendimiento } \\
\text { Boyacá (t ha-1) }\end{array}$ & 24,5 & 24 & 26 & 25,7 & 26,2 & 23,3 & 23,4 & 27,6 & 26,3 & 27,8 & 24,7 & 32,1 \\
\hline $\begin{array}{l}\text { Rendimiento } \\
\text { Colombia (t ha-1) }\end{array}$ & 19,9 & 19,8 & 21,9 & 22,2 & 21,8 & 21,1 & 21,1 & 21,6 & 21,4 & 21,6 & 19,7 & 16,8 \\
\hline
\end{tabular}

Fuente: Elaborado con base en datos de Agronet, 2009.

Tabla 5. Correlación entre los parámetros climáticos de la estación Surbatá/Bonza del municipio Duitama (Boyacá) y la producción anual del departamento Boyacá (1997-2008).

\begin{tabular}{|c|c|c|c|c|c|c|c|c|c|}
\hline Coeficiente & Brillo solar & Evaporación & $\begin{array}{l}\text { Humedad } \\
\text { relativa }\end{array}$ & Precipitación & $\begin{array}{l}\text { Precipitación } \\
\text { máx. en } 24 \text { h }\end{array}$ & No. de días & $\begin{array}{c}\text { Tempe- } \\
\text { ratura } \\
\text { media } \\
\text { anual } \\
\end{array}$ & $\begin{array}{c}\text { Tempe- } \\
\text { ratura } \\
\text { máx. anual }\end{array}$ & $\begin{array}{c}\text { Tempe- } \\
\text { ratura } \\
\text { mín. anual }\end{array}$ \\
\hline & (h año-1) & $(\mathrm{mm}$ año-1) & $\begin{array}{l}\text { promedio } \\
\text { anual }(\%)\end{array}$ & (mm año-1) & $(\mathrm{mm})$ & $\begin{array}{c}\text { con lluvia/ } \\
\text { año }\end{array}$ & $\left({ }^{\circ} \mathrm{C}\right)$ & $\left({ }^{\circ} \mathrm{C}\right)$ & $\left({ }^{\circ} \mathrm{C}\right)$ \\
\hline $\mathrm{R}^{2}$ & 0,3725 & 0,8171 & 0,0666 & 0,1574 & 0,0384 & 0,052 & 0,0757 & 0,0011 & 0,1198 \\
\hline Relación* & directa & inversa & directa & directa & inversa & directa & directa & directa & inversa \\
\hline
\end{tabular}

Fuente: Elaborado con base a datos del Ideam, 2009 y Agronet, 2009.

* Relación directamente proporcional o relación inversamente proporcional. 
dos a través del manejo del cultivo: semilla, épocas de siembra, riego, control de plagas, enfermedades y malezas.

Según los productores entrevistados, los factores que más han incidido en el incremento o disminución de cultivos de cebolla en la zona han sido los precios y las enfermedades (tizón y alternaria). Según las fuentes primarias, a finales de 2007 los precios llegaron hasta 5.000 \$/bulto, así como a finales de 2008 llegaron a $140.000 \$ /$ bulto. La caída de los precios se dio por la abundante producción (clima favorable y expectativas de los productores) y por la importación de cebolla. El ataque de las enfermedades proliferó en los últimos dos años. Este incremento se atribuye a dos factores: incremento de fuentes de inóculo (más cultivos con enfermedad) y condiciones ambientales favorables (mayor humedad por efecto del riego y precipitaciones y mayores temperaturas).

\section{Medidas adoptadas por los productores para contrarrestar los efectos del cambio climático en la producción de cebolla de bulbo}

Frente a los problemas de precios bajos y ataque de enfermedades que se han presentado en los últimos años, los productores de cebolla que han permanecido en el negocio han adoptado, casi todos, las siguientes medidas:

- Tratar de utilizar semilla certificada. Antes se utilizaban hasta 15 libras por hectárea, ahora aproximadamente 8 libras/hectárea.

- Escalonar las épocas de siembra.

- Incrementar la frecuencia de fumigado contra enfermedades. Antes cada 10-12 días, ahora cada 3-7 días. Otros productores dicen que ahora se fumiga de 9 a 15 veces por ciclo.

- Incrementar las dosis de fungicidas (casi el doble de lo que se utilizaba antes).

- Reducir la superficie de cultivo. En algunos casos se redujo hasta un $50 \%$.
Lógicamente, el incremento de la frecuencia de aplicaciones y dosis de fungicidas ha incrementado los costos de producción, tanto por producto comprado como por mano de obra utilizada. A diferencia de los datos provenientes del Banco Agrario (2008), el técnico entrevistado sostenía que los costos de producción de cebolla ascendía a 18.000.000 \$/ha, de los cuales alrededor del $30 \%$ correspondían a controles fitosanitarios.

Algo que llamó la atención fue que la mayoría de los productores no han intentado mejorar el manejo del riego, las densidades de siembra y el monitoreo de enfermedades. Sin embargo, los productores más grandes ya lo están haciendo, por ejemplo, a través de aplicación de riegos por demanda del cultivo y no por rutina, disminución de densidades de siembra a 3 libras/fanegada y monitoreos del ataque de enfermedades antes de fumigaciones rutinarias.

\section{Efectos de la producción en la oferta (producción nacional e importación) y precios de la cebolla de bulbo}

Si bien la producción nacional cubre cerca del $80 \%$ de la oferta nacional de cebolla de bulbo (año 2008), la importación está cobrando importancia (tabla 6). Mientras la producción nacional tiene una tendencia de crecimiento de $31 \%$, la de importación es de $355 \%$. Por el contrario, las exportaciones de cebolla tienen una tendencia de disminución del 87\%. La producción de Boyacá, que aporta aproximadamente el $50 \%$ a la producción colombiana, tiene gran influencia en la oferta nacional de cebolla.

Los precios nominales de la cebolla de bulbo han tenido una tendencia al incremento en una proporción del 64\% en los últimos 12 años (tabla 6). Relacionando precios y producción (oferta nacional), se ha encontrado una baja correlación $(<0,2)$ entre estas dos variables, de lo que podría deducirse una baja influencia de solamente la producción sobre los precios y viceversa (Corabastos, 2009; Agronet, 2009). Sin embargo, se advierte una creciente (aproximadamente 5\% 
Tabla 6. Oferta nacional y precios mayoristas (Corabastos, Bogotá) de cebolla de bulbo en Colombia (1997-2008).

\begin{tabular}{|c|c|c|c|c|c|c|c|c|c|c|c|c|}
\hline Variable & 1997 & 1998 & 1999 & 2000 & 2001 & 2002 & 2003 & 2004 & 2005 & 2006 & 2007 & 2008 \\
\hline $\begin{array}{l}\text { Producción } \\
\text { nacional (t) }\end{array}$ & 230.595 & 203.410 & 257.099 & 276.300 & 224.945 & 216.558 & 232.817 & 251.752 & 253.728 & 294.790 & 312.673 & 277.152 \\
\hline Importación (t) & 11.028 & 14.154 & 21.005 & 38.333 & 30.936 & 35.620 & 34.538 & 44.436 & 43.990 & 51.449 & 44.713 & 71.826 \\
\hline Exportación (t) & -3.090 & -53 & -3.657 & -20.396 & -2.024 & -619 & -340 & -910 & -990 & -1.517 & -1.626 & -1.328 \\
\hline $\begin{array}{l}\text { Oferta para } \\
\text { Colombia (t) }\end{array}$ & 238.533 & 217.511 & 274.447 & 294.237 & 253.857 & 251.559 & 267.015 & 295.278 & 296.728 & 344.722 & 355.760 & 347.650 \\
\hline $\begin{array}{l}\text { Precios } \\
\text { mayoristas } \\
(\$ / \mathrm{kg})\end{array}$ & 511 & 829 & 478 & 854 & 474 & 792 & 812 & 852 & 780 & 861 & 831 & 1063 \\
\hline
\end{tabular}

Fuente: Elaborado con base en datos de Agronet, 2009 y Corabastos, 2009.

anual) importación de cebolla proveniente de Ecuador (77\% el año 2008), Perú (19\% el año 2008), Holanda (2\% el año 2008) y Chile $(<1 \%$ el año 2008) (Agronet, 2009). El comportamiento de la importación de cebolla tiene una correlación del 0,5 con el comportamiento de los precios en las centrales mayoristas como Corabastos de Bogotá, lo que hace pensar que la importación está influyendo en la formación de los precios de cebolla mucho más que la producción local y nacional. Así lo manifestaron también los productores entrevistados, quienes aducían a la importación la caída de los precios y la disminución de la superficie de cultivo de la cebolla.

\section{CONCLUSIONES}

- El cambio climático es un fenómeno que ya se ha hecho presente en la zona de estudio. Se caracteriza principalmente por el incremento de la temperatura media, de la evaporación, de la precipitación (menor frecuencia y mayor intensidad) y por una disminución del brillo solar y de la humedad relativa. Además, los rangos entre máximos y mínimos de la mayoría de los parámetros climáticos tienen una tendencia a ampliarse.

- El cambio climático, según los entrevistados, ha incidido en los cultivos de cebolla de la zona de estudio, pero no ha ocasionado grandes cambios en la cantidad de producción.
- Los efectos más notorios de los cambios en el clima se han manifestado, según los productores, con incrementos del ataque de enfermedades (tizón y alternaria) y mayor necesidad de riego para el cultivo. Siendo éste el efecto más visible, no significa, sin embargo, que problemas como ataque de plagas y presión de malezas al cultivo no estén asociados al cambio climático.

- Las medidas adoptadas por los productores frente al fenómeno del cambio climático, de una manera general, fueron el incremento de la frecuencia $\mathrm{y}$ dosis de las fumigaciones contra enfermedades y el incremento de la frecuencia de riegos. Estas medidas no son suficientes, y en muchos casos no son las más apropiadas, teniendo en cuenta que hay medidas de manejo integral del cultivo que podrían hacer más eficiente el uso de recursos (entre otros, agua, fungicidas y mano de obra) durante el proceso de producción.

- Los productores grandes, a diferencia de los medianos y pequeños, son los que están adoptando otras medidas, como el manejo de las densidades y del riego, así como el monitoreo de enfermedades.

- El cambio climático, a través de una mayor o menor producción, no afecta el precio de la cebolla de manera significativa, su efecto se manifiesta más en los costos de producción y en la ganancia de los productores. Las importaciones y las expectativas (de una mejor ganancia) 
de los productores han resultado ser los factores que más determinan los cambios de precios de la cebolla de bulbo.

- Este estudio exploratorio da pie para profundizar en temas específicos como: influencia del cambio climático en el incremento de problemas fitosanitarios del cultivo de cebolla, respuesta estratégica de los productores de cebolla al cambio climático e introducción de nuevas estrategias de transferencia de tecnología.

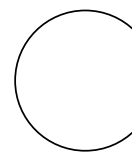

AGRADECIMIENTOS

Los autores expresan su agradecimiento al Instituto Colombiano Agropecuario (ICA), seccional Boyacá, al Instituto de Hidrología, Meteorología y Estudios Ambientales (Ideam), estación Surbatá-Bonza, Duitama (Boyacá) y a los productores de cebolla entrevistados por la información brindada. Un especial agradecimiento al Distrito de Riego y Drenaje de Gran Escala Alto Chicamocha y Firavitoba (Usochicamocha), que por medio de los señores Horacio Pachón y Hector Centeno nos facilitó el recorrido por la zona de estudio y el contacto con los productores de cebolla.

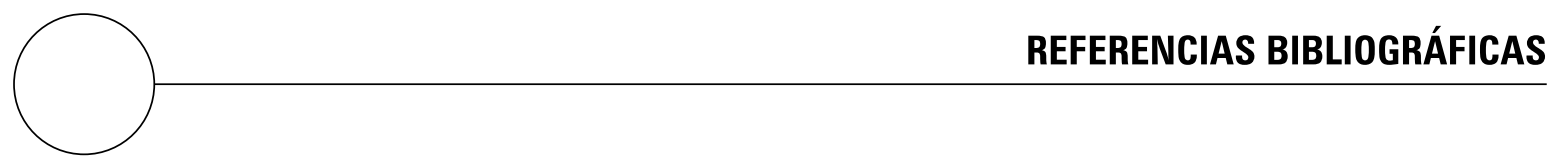

Agrios, G. 2005. Plant pathology. $5^{\text {th }}$ ed. Elsevier Academic Press, Burlington, MA.

Agronet. 2009a. Encuesta Nacional Agropecuaria 20012008. En: Agronet, http://www.agronet.gov.co/ agronetweb/Boletines/tabid/75/Default.aspx; consulta: mayo de 2009.

Agronet. 2009b. Área, rendimiento y producción. Cebolla cabezona. En: Agronet, http://www.agronet.gov.co; consulta: junio de 2009 .

Agronet. 2009c. Importaciones de Cebolla fresca. En: Agronet, http://www.agronet.gov.co; consulta: junio de 2009.

Banco Nacional Agrario de Colombia. 2008. Cultivo cebolla cabezona - Boyacá (Allium cepa L.). Costo de producción por cosecha/hectárea. En: http:// www.bancoagrario.gov.co/webapp/PaintServle t? . node $=003007 \&$ tree ManagerId $=1 \&$ tree $I d=1$; consulta: junio de 2009.

Brewster, J. 1997. Onions and garlic. pp. 581-619. En: Wien, $\mathrm{H}$. (ed.). The physiology of vegetable crops. CABI, Wallingford, UK.

CCI. 2003. Monitoreo de mercados: Mercado nacional de frutas y hortalizas No. 5. junio de 2003. Corporacion Colombia Internacional, Bogotá.

CCI. 2009. Encuesta Nacional Agropecuaria 2001-2008. En: CCI, http://www.cci.org.co/cci/cci_x/scripts/ home.php? men $=226 \& \mathrm{con}=70 \& \mathrm{rdH} \mathrm{m}=2 \& \mathrm{opc}=9$ 9; consulta: mayo de 2009

Corporación de Abastos de Bogotá S.A. (Coraboastos, Mercado mayorista). 2009. Información anual de precios de mercado mayorista. Calidad extra Cebolla cabezona blanca. Bogotá.

Distrito de Riego y Drenaje de Gran Escala Alto Chicamocha y Firavitoba (Usochicamocha) 2008. Presentación de la institución (ppt). Duitama, Boyacá.

Ezekiel, M. 1938. The cobweb theorem. Quarterly Journal of Economics 52: 255-280. Cambridge. Traducción al castellano: El teorema de la telaraña; Gottfried Haberler (ed.) 1944. Ensayos sobre el ciclo económico. pp. 437-457. Fondo de Cultura Económica, México.

Instituto de Hidrología, Meteorología y Estudios Ambientales (Ideam). 2009. Sistema de información nacional ambiental. Bogotá.

Kurukulasuriya, P. y S. Rosenthal. 2003. Climate change and agriculture: A review of impacts and adaptations. Paper No. 91 in Climate Change Series, Ariculture and Rural. Development Department and Environment Department, World Bank, Washington, DC.

Maroto, J.; B. Pascual y V. Borrego. 1995. Enfermedades de las hortalizas. Ediciones Mundi-Prensa, Madrid. pp. 361-400. 